\title{
Three-dimensional morphometric differences of resected distal femurs and proximal tibias in osteoarthritic and normal knees
}

\author{
Xiang-hui Dong ${ }^{1,2}$, Xiang-hui Huang ${ }^{1}$, Ming Chen ${ }^{1}$, Yan-Hai Chang ${ }^{1}$, Ming Ling ${ }^{1}$ and Bo Yang ${ }^{1 *}$
}

\begin{abstract}
Background: There is a paucity of data concerning the morphological differences of resected distal femurs and proximal tibias in osteoarthritic $(\mathrm{OA})$ and normal knees. The objective of this study was to determine whether morphometric differences in the surfaces of resected distal femurs and proximal tibias exist between OA and normal knees in a Chinese population.

Methods: Ninety-four OA knees and ninety-five normal knees were evaluated in Chinese individuals. Computed tomography was used to measure the femoral mediolateral (fML), medial anteroposterior (fMAP), lateral anteroposterior (fLAP), medial condylar width (fMCW), lateral condylar width (fLCW), medial posterior condylar curvature radii ( $\mathrm{fMCR}$ ), lateral posterior condyle curvature radii ( $\mathrm{fLCR}$ ), fML/fMAP aspect ratio, tibial mediolateral (tML), middle anteroposterior (tAP), medial anteroposterior (tMAP), and lateral anteroposterior (tLAP) tML/tMAP aspect ratio to determine the morphologic differences between $O A$ and normal knees.

Results: The average fMCW and tMAP dimensions of OA knees were larger than those of normal knees in both male and female $(p<0.05)$. The $\mathrm{fMAP} / \mathrm{fML}$ aspect ratio and tMAP/tML aspect ratio were also significantly different in both sexs $(p<0.05)$. OA knees have an oval-shaped distal femur with a wider ML length and more spherical-shaped proximal tibiae with relatively narrow ML dimensions.

Conclusions: The study revealed the morphological differences in $\mathrm{FMCW}$, tMAP, $\mathrm{FMAP/fML}$ and $\mathrm{MMAP} / \mathrm{tML}$ between $\mathrm{OA}$ and normal knees in both males and females. These findings may provide guidelines that can be used to design better knee implants that are more size-matched for OA knees.
\end{abstract}

Keywords: Computed tomography, Osteoarthritis, Normal knee, Total knee arthroplasty, Morphometry

\section{Background}

An appropriate prosthesis size that matches the resected bony surfaces is considered a crucial factor for success in total knee arthroplasty (TKA) [1, 2]. If the prosthesis

\footnotetext{
*Correspondence: yangbo1981911@126.com

1 Department of Orthopaedics, Shaanxi Provincial People's Hospital (third affiliated hospital of Xi'an jiaotong University), No. 256, Youyi West Road, Xi'an, China

Full list of author information is available at the end of the article
}

underhangs the resected surface of the bone, it may cause early subsidence and loosening of the prosthesis, whereas an overhang may cause residual pain, poor knee flexion, and decreased functional results [3, 4]. Thus, it is important to maximize coverage of the knee component on the resected bony surface to ensure a good clinical result and long-term survivorship of the prosthesis $[5,6]$. To design a proper knee component, many researchers have measured the resected surface of normal knees from imaging original author(s) and the source, provide a link to the Creative Commons licence, and indicate if changes were made. The images or other third party material in this article are included in the article's Creative Commons licence, unless indicated otherwise in a credit line to the material. If material is not included in the article's Creative Commons licence and your intended use is not permitted by statutory regulation or exceeds the permitted use, you will need to obtain permission directly from the copyright holder. To view a copy of this licence, visit http://creativecommons.org/licenses/by/4.0/. The Creative Commons Public Domain Dedication waiver (http://creativecommons.org/publicdomain/zero/1.0/) applies to the data made available in this article, unless otherwise stated in a credit line to the data. 
data $[7,8]$, while others have analyzed anthropometric features of diseased knees during TKA $[9,10]$. It is unclear whether there are morphometric differences in the resected bony surfaces between diseased and normal knees.

Generally, most knees that undergo TKA are deformed and shaped differently than healthy knees. This suggests that the design of the prosthesis should be based on data from diseased knees [11]. However, most of the currently available TKA prostheses are designed based on the anthropomorphic features of normal knees [12]. Such prostheses may not necessarily provide the best fit for TKA candidates. Osteoarthritis accounted for more than $90 \%$ of the patients who underwent primary TKA $[13,14]$. To the best of our knowledge, no morphometric differences in the resected distal femur and proximal tibia surface between the OA and normal knees have been compared. The aim of this study, therefore, was to measure the morphometric features of the resected distal femur and proximal tibia surface to determine whether there are morphometric differences between $\mathrm{OA}$ and normal knees.

\section{Methods}

This study was performed with the informed consent of each subject and approved by the institutional review board of Shaanxi Provincial People's Hospital. In this study, the morphology of 94 (49 males and 45 females) OA knees that were candidates for TKA and 95 (48 males and 47 females) normal knees after anterior cruciate ligament construction or knee trauma without fracture, congenital anomalies or pathological deformities around the knee joint were recorded from June 2017 to April 2018. According to the Kellgren and Lawrence classification, all OA knees had radiographic evidence of grade III-IV osteoarthritis. There were 12 grade III and 37 grade IV in males and 9 grade III and 36 grade IV in females.

Computer tomography (CT) imaging was performed using a helical CT scanner imaging machine $(120 \mathrm{kVp}$, $200 \mathrm{~mA}$, Somatom Sensation, Siemens Health care, Germany). The subjects were placed in the supine position on the scanner with knees in the full extended position and their patella facing toward the ceiling. The scanning procedure was performed to acquire $1.0 \mathrm{~mm} \mathrm{CT}$ slices (image size: $512 \times 512$ pixels). In the CT scan, a 15 $\mathrm{cm}$ femur and tibia diaphysis were included. The images of the knees were segmented using a region-growing method to construct 3D bony models by Mimics 17.0 software (Belgium, Materialise). The measurements were performed using Geomagic Studio 14.0 software (USA, Raindrop).

A point was marked in the center of the femoral intramedullary canal $12 \mathrm{~cm}$ from the distal femoral joint surface. A line connecting this point and the point $5 \mathrm{~mm}$ anterior to the intercondylar notch apex was defined as the femoral anatomic axis. The distal femur was cut 9 $\mathrm{mm}$ above the lowest point of the medial condyle with $6^{\circ}$ valgus to the anatomical axis (Fig. $1 \mathrm{a}$ ). A line connecting the medial sulcus (the insertion point of the deep fibers of the medial collateral ligament )[15] of the medial epicondyle and the lateral epicondylar prominence was defined as the surgical transepicondylar axis (STEA). The femoral mediolateral (FML) dimension was defined as the longest ML length of the distal cut femur surface; this line paralleled the STEA. The femoral lateral anteroposterior (fLAP) and medial anteroposterior (fMAP) dimensions were defined as the longest line drawn perpendicular to the fML between the most posterior condylar and the anterior trochlear point from the lateral and medial condyle of the femur. The medial and lateral condyle widths were measured $10 \mathrm{~mm}$ above the lowest point of the

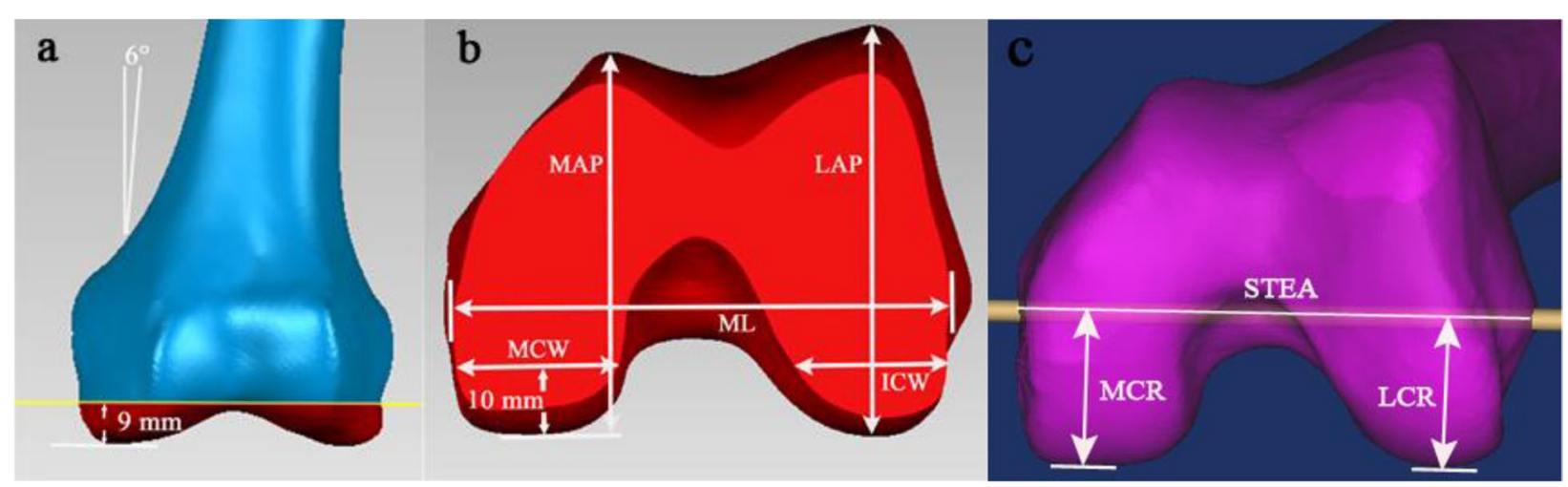

Fig. 1 Distal femur resection and measurement on CT images. a Resection method of distal femur. b Measurements of resected femoral surfaces. $\mathbf{c}$ Measurements of femoral posterior condyle curvature radii 
medial posterior condyles to simulate the optimal cutting thickness (Fig. 1 b). The femoral medial posterior condyle curvature radii (fMCR) and lateral posterior condyle curvature radii (fLCR) were defined as the vertical distance between the STEA (functional flexion-extension axis) and the most posterior point of the medial and lateral posterior condyles, respectively (Fig. $1 \mathrm{c}$ ).

A point was marked in the center of the tibial intramedullary canal $12 \mathrm{~cm}$ from the proximal tibial joint surface. A line connecting this point and the center of the tibial spines was defined as the tibial mechanical axis. The proximal tibial cut was performed perpendicular to the mechanical axis of the tibia, $8 \mathrm{~mm}$ below the lateral tibial plateau with $5^{\circ}$ of posterior inclination (Fig. 2 a). The tibial mediolateral (tML) dimension was taken as the longest mediolateral length of the resected tibial surface. This line is parallel to the surgical epicondylar axis of the femur and formed by connecting the medial sulcus of the medial epicondyle and the lateral epicondylar prominence. The tibial middle anteroposterior (tAP) dimension was taken as the length of the line drawn perpendicular and passing through the midpoint of the tML line. The tibial lateral anteroposterior (tLAP) and medial anteroposterior (tMAP) dimensions were taken as the length of the line drawn perpendicular to the tML and passing through the posterior-most point of the lateral and medial tibial condyle (Fig. 2 b). The condylar aspect ratios of fMAP to fML (fMAP/fML) and the plateau aspect ratio of tMAP to tML (tMAP/tML) were calculated as described by Hitt [16].

\section{Statistical analysis}

SPSS software 18.0 (SPSS, Chicago, IL) was used for statistical analysis. The mean and standard deviation of the measured dimensions were calculated. A Kolmogorov-Smirnov test for normality was performed, and the data were found to be normally distributed. Independent sample t-tests were used to determine the significance of morphological differences between OA and normal knees. To determine the influence of height on the knee joint dimensions, all data were additionally corrected for body height. The differences were regarded as significant when $p<0.05$.

\section{Results}

OA subjects were older, lower and had larger HKA angles than normal knee subjects. There was a significant difference in the body weight of female subjects $(p<0.01)$. The demographic characteristics of the subjects are summarized in Table 1.

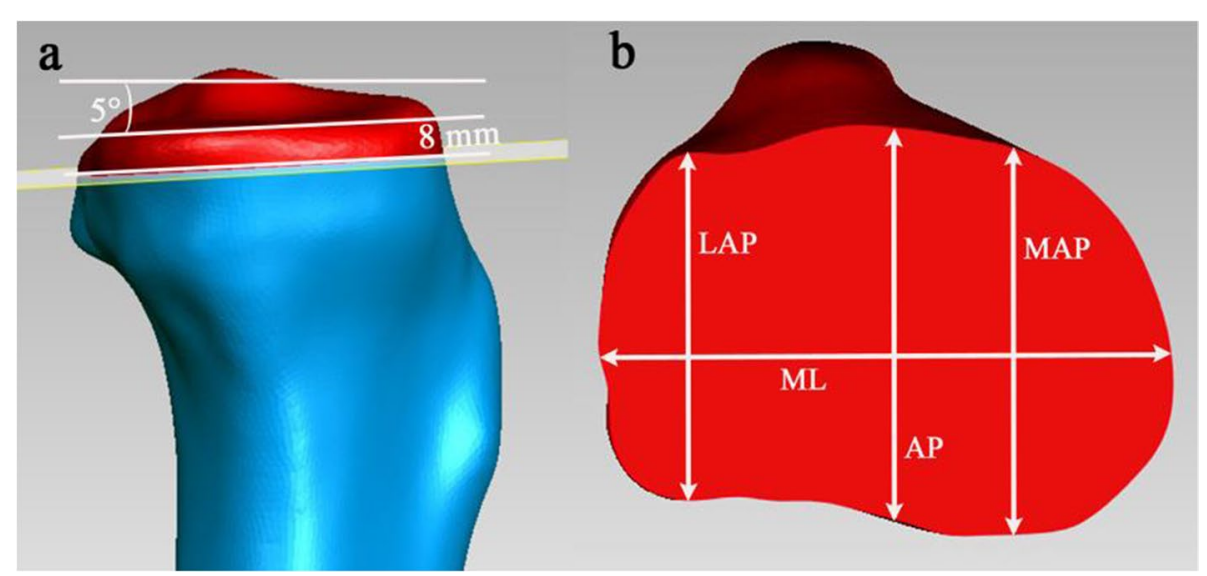

Fig. 2 Proximal tibia resection and measurement on CT images. a Resection method of proximal tibia. b Measurements of resected tibial surfaces

Table 1 The demographic data of subjects

\begin{tabular}{|c|c|c|c|c|c|c|}
\hline \multirow[t]{2}{*}{ Parameter } & \multicolumn{3}{|l|}{ Male } & \multicolumn{3}{|l|}{ Female } \\
\hline & $O A$ & Normal & $p$ & $O A$ & Normal & $p$ \\
\hline Age (year) & $64.6 \pm 6.2$ & $28.8 \pm 5.7$ & $<0.01$ & $65.5 \pm 5.9$ & $31.1 \pm 7.0$ & $<0.01$ \\
\hline Body weight (kg) & $75.7 \pm 9.8$ & $75.3 \pm 10.5$ & 0.886 & $67.0 \pm 8.4$ & $66.6 \pm 9.8$ & 0.815 \\
\hline Height (cm) & $170.2 \pm 7.3$ & $175.1 \pm 6.5$ & $<0.01$ & $160.5 \pm 5.8$ & $164.7 \pm 6.2$ & $<0.01$ \\
\hline $\operatorname{HKA}\left(^{\circ}\right)$ & $6.8 \pm 3.2$ & $0.8 \pm 1.9$ & $<0.01$ & $8.3 \pm 2.1$ & $1.4 \pm 2.3^{\circ}$ & $<0.01$ \\
\hline
\end{tabular}


On the basis of the numbers available, the average fMCW dimensions $(28.9 \pm 2.8 \mathrm{~mm}$ for males and $27.3 \pm 2.4 \mathrm{~mm}$ for females) of OA knees were larger than the dimensions $(27.0 \pm 2.9 \mathrm{~mm}$ for males and $25.4 \pm 2.3$ $\mathrm{mm}$ for females) of normal knees $(p<0.01)$. The condylar aspect ratio (fMAP/fML) was also significantly different $(p<0.05)$. After correcting for body height, there was a significant difference in $\mathrm{fMCW}$ and $\mathrm{fMAP} / \mathrm{fML}$ in both males and females $(p<0.05)$. Morphological differences found between the OA and normal groups in terms of distal femur dimensions are summarized in Table 2.

The average tMAP dimensions and plateau aspect ratio (tMAP/tML) showed significant differences between OA and normal knees in both males and females $(\mathrm{p}<0.05)$. After correcting for body height, there was also a significant difference in tMAP and $\mathrm{PMAP} / \mathrm{fML}$ in both sexes $(\mathrm{p}<0.05)$ (Table 3).

\section{Discussion}

In this study, we measured the morphology of the resected distal femur and proximal tibia surfaces of OA and normal knees. The major findings were that the fMCW and tMAP dimensions in OA subjects were significantly larger than those of normal knees. In a study by Puthumanapully et al., the authors found that varus knees had larger femur dimensions of the medial condyle than normal knees [17]. The morphological differences in the medial condyle between OA and normal knees may be explained by the pathological changes in OA knees. Most OA knees of TKA candidates had varus deformity, and the medial condyle experienced destruction and remodeling in response to larger loads during gait [18], which could eventually result in bony structural changes in the medial condyle of OA knees. In addition, there was a difference in the distal femur condyle and proximal tibia plateau aspect ratio between OA and normal knees. OA knees were found to have an oval-shaped distal femur with a wider ML length and more spherical-shaped proximal tibiae with relatively narrow ML dimensions.

Optimal coverage of the component to the resected bony surface is essential for long-term good outcomes after TKA. If the implant mismatches the resected bone surface, there will be undersizing or overhang, which could result in worse clinical outcomes [19]. Thus, it is critical to design a size-matching component for TKA candidates according to knee morphology. Various morphological studies of resected bony surfaces from normal $[7,20]$ or OA knees $[9,21]$ have been conducted to provide data for proper size matching. Cheng et al. [11] suggested that the design of the knee component should be based on data from diseased knees rather than normal knees. To date, no studies have examined the morphological differences of resected femoral and tibial surfaces between diseased and normal knees to determine which

Table 2 Distal femur dimensions in OA and normal knees ( $\mathrm{mm}$ )

\begin{tabular}{|c|c|c|c|c|c|c|c|c|}
\hline \multirow[t]{2}{*}{ Parameter } & \multicolumn{4}{|l|}{ Male } & \multicolumn{4}{|l|}{ Female } \\
\hline & $O A$ & Normal & $p$ & Corrected $p$ & $\mathrm{OA}$ & Normal & $p$ & Corrected $p$ \\
\hline $\mathrm{fML}$ & $76.8 \pm 3.5$ & $76.3 \pm 3.2$ & 0.493 & 0.121 & $71.4 \pm 4.3$ & $70.7 \pm 3.2$ & 0.401 & 0.136 \\
\hline fMAP & $64.0 \pm 3.7$ & $64.7 \pm 2.7$ & 0.267 & 0.262 & $59.8 \pm 3.2$ & $60.5 \pm 3.6$ & 0.320 & 0.188 \\
\hline$f L A P$ & $66.7 \pm 3.8$ & $67.3 \pm 3.3$ & 0.431 & 0.193 & $61.4 \pm 3.1$ & $61.7 \pm 4.8$ & 0.790 & 0.239 \\
\hline fMCW & $28.9 \pm 2.8$ & $27.3 \pm 2.4$ & 0.018 & $<0.01$ & $27.0 \pm 2.9$ & $25.4 \pm 2.3$ & $<0.01$ & $<0.01$ \\
\hline $\mathrm{fLCW}$ & $26.8 \pm 2.9$ & $26.4 \pm 2.7$ & 0.299 & 0.062 & $24.6 \pm 2.1$ & $24.2 \pm 2.0$ & 0.358 & 0.103 \\
\hline $\mathrm{fMCR}$ & $23.7 \pm 2.1$ & $24.2 \pm 2.3$ & 0.392 & 0.061 & $21.6 \pm 2.0$ & $22.2 \pm 2.2$ & 0.296 & 0.290 \\
\hline$f L C R$ & $22.1 \pm 2.1$ & $22.3 \pm 1.8$ & 0.610 & 0.293 & $20.5 \pm 2.2$ & $20.9 \pm 2.2$ & 0.356 & 0.537 \\
\hline fMAP/fML & $0.83 \pm 0.03$ & $0.85 \pm 0.03$ & 0.028 & 0.028 & $0.84 \pm 0.04$ & $0.86 \pm 0.03$ & 0.027 & 0.028 \\
\hline
\end{tabular}

Table 3 Proximal tibia dimensions in $\mathrm{OA}$ and normal knees $(\mathrm{mm})$

\begin{tabular}{|c|c|c|c|c|c|c|c|c|}
\hline \multirow[t]{2}{*}{ Parameter } & \multicolumn{4}{|l|}{ Male } & \multicolumn{4}{|l|}{ Female } \\
\hline & $O A$ & Normal & $p$ & Corrected $p$ & $O A$ & Normal & $p$ & Corrected $p$ \\
\hline $\mathrm{tML}$ & $78.6 \pm 3.5$ & $78.8 \pm 3.2$ & 0.836 & 0.148 & $72.2 \pm 3.2$ & $71.9 \pm 3.1$ & 0.651 & 0.135 \\
\hline tMAP & $55.0 \pm 3.1$ & $53.7 \pm 2.1$ & 0.017 & $<0.01$ & $50.6 \pm 2.7$ & $48.9 \pm 2.3$ & 0.002 & $<0.01$ \\
\hline tAP & $52.7 \pm 3.2$ & $52.3 \pm 3.0$ & 0.371 & 0.054 & $47.7 \pm 2.7$ & $47.4 \pm 2.2$ & 0.570 & 0.116 \\
\hline tLAP & $50.7 \pm 3.6$ & $50.1 \pm 3.3$ & 0.382 & 0.067 & $45.6 \pm 3.0$ & $45.3 \pm 2.6$ & 0.529 & 0.087 \\
\hline $\mathrm{tMAP} / \mathrm{fML}$ & $0.70 \pm 0.03$ & $0.68 \pm 0.02$ & $<0.01$ & $<0.01$ & $0.70 \pm 0.02$ & $0.68 \pm 0.03$ & 0.027 & 0.027 \\
\hline
\end{tabular}


morphological data are more suitable to use to design proper components.

Many studies have reported on the measurements of resected proximal tibia surfaces in Asian knees. Cheng et al. [7] reported mean tML, tAP, tMAP, and tLAP values were $76.4 \mathrm{~mm}, 51.3 \mathrm{~mm}, 53.3 \mathrm{~mm}, 47.7 \mathrm{~mm}$ for male and $68.8 \mathrm{~mm}, 45.7 \mathrm{~mm}, 47.5 \mathrm{~mm}, 42.4 \mathrm{~mm}$ for female in 172 Chinese normal tibias by CT imaging. Kwak et al. [22] studied 200 normal cadaver tibias and determined that the tML, tAP, tMAP, and tLAP values were $76.1 \mathrm{~mm}, 48.2$ $\mathrm{mm}, 48.8 \mathrm{~mm}$, and $44.6 \mathrm{~mm}$ for males and $67.6 \mathrm{~mm}, 43.2$ $\mathrm{mm}, 43.5 \mathrm{~mm}$, and $39.8 \mathrm{~mm}$ for females, respectively, on CT imaging. Karimi et al. [5] studied 132 normal tibias from the Iran population on MRI scans and determined that the tML, tAP, tMAP, and tLAP values were $77.8 \mathrm{~mm}$, $48.8 \mathrm{~mm}, 53.1 \mathrm{~mm}$, and $52.0 \mathrm{~mm}$ for males and $66.5 \mathrm{~mm}$, $43.1 \mathrm{~mm}, 45.5 \mathrm{~mm}$, and $43.7 \mathrm{~mm}$ for females. The results in our Chinese subjects were slightly larger than those in the overall Asian population, which might be due to the difference in imaging techniques and the difference in the heights of the individuals in the study group. In addition, the depth of the resection affects the sizing of the resected bony surface. The depth of the proximal tibia resection in our study at a thickness of $8 \mathrm{~mm}$ below the lateral tibial plateau was higher than the $10 \mathrm{~mm}$ [5] or lower than the $6 \mathrm{~mm}$ [22] depth used in other studies.

Several researchers have studied the dimensions of distal femurs in Asian populations. Cheng et al. [7] reported the mean $\mathrm{fML}$ and fLAP values on CT to be $74.4 \mathrm{~mm}$ and $66.6 \mathrm{~mm}$ for males and $66.8 \mathrm{~mm}$ and $61.0 \mathrm{~mm}$ for females, respectively, in normal Chinese femurs. Lim et al. [20] showed that femoral fML, fMAP and fLAP dimensions were $81.5 \mathrm{~mm}, 62.7 \mathrm{~mm}, 59.0 \mathrm{~mm}$ for males and $76.7 \mathrm{~mm}, 56.8 \mathrm{~mm}, 58.4 \mathrm{~mm}$ for females in a Korean population using MRI. Urabe et al. [23] studied the distal femur using CT imaging in a Japanese population and reported fML, $\mathrm{AMCW}$ and $\mathrm{FLCW}$ dimensions of 70.6 $\mathrm{mm}, 30.1 \mathrm{~mm}$ and $24.8 \mathrm{~mm}$ in OA subjects. Vaidya et al. [24] used CT to show that femoral ML and LAP dimensions were $68.8 \mathrm{~mm}$ and $56.6 \mathrm{~mm}$ in males and $64.1 \mathrm{~mm}$ and $56.8 \mathrm{~mm}$ in females in Indian OA knees. Our results showed minor differences between these Asian populations. which might be due to the difference between the heights of the study groups. Charlton et al. [25] reported a significant difference in the femoral bicondylar width between short and tall subjects, with taller subjects having larger values.

To date, many studies have confirmed knee anatomic differences in Caucasian and Asian populations $[26,27]$. However, nearly all existing TKA components were designed based on the anatomy of Caucasian populations and are not suitable for Asian patients [7, $28]$. In the clinic, Iorio et al. followed (9 vs. 6.6 years)
American and Japanese patients after primary TKA and showed that American patients required significantly larger implants than Japanese patients. The authors also found that Japanese patients had significantly less postoperative range of motion (93.7 vs. $106.6^{\circ}$ ) and a higher revision rate $(4.1 \%$ vs. $2.6 \%)$ than American patients [29]. Anatomy studies and clinical outcomes all demonstrated that ethnic differences should be considered when designing proper TKA components for Asian populations.

We acknowledge that this study included a limited number of subjects and may not adequately reflect the features of $\mathrm{OA}$ and normal knees. If a larger sample size was included in the study, other significant differences may be noticed. We are also aware that only one bone resection level was measured. However, resection depth varies according to the stage of disease during TKA. In the future, we will report data for a larger sample size and measure the depth at different resection levels.

\section{Conclusion}

In summary, our study demonstrated that the $\mathrm{fMCW}$, tMAP dimensions, and MAP/ML aspect ratio of the distal femur condylar and proximal tibia plateau of resected knee surfaces were indeed significantly different in OA knees than in normal knees. As a result, we believe that the shape variations of the OA knees and normal subjects should be a concern when designing components for TKA candidates.

\section{Abbreviations}

OA: Osteoarthritis; tML: Tibial mediolateral; tAP: Tibial middle anteroposterior; tMAP: Tibial medial anteroposterior; tLAP: Tibial lateral anteroposterior; fML: Femoral mediolateral; fMAP: Femoral medial anteroposterior; fLAP: Femoral lateral anteroposterior; fMCW: Femoral medial condylar width; fLCW: Femoral lateral condylar width; fMCR: Femoral medial posterior condyle curvature radii; fLCR: Femoral lateral posterior condyle curvature radii; TKA: Total knee arthroplasty; CT: Computer tomography; STEA: Surgical transepicondylar axis; BMI: Body mass index; HKA: Hip knee ankle.

\section{Acknowledgements}

Not applicable.

\section{Authors' contributions}

XHD designed the study, analyzed the data, wrote the manuscript. BY designed the study, analyzed the data and revised the manuscript. XHH and MC performed measurements and analysis the data. YHC and ML designed the study and reviewed the manuscript. All authors read and approved the final content of the manuscript.

\section{Funding}

This research was supported by Shaanxi provincial people's hospital incubation fund (No.2019YXQ-13). International science and technology cooperation and exchanges (No.2019KW-070).

\section{Availability of data and materials}

The datasets used and/or analyzed during the present study are available from the corresponding author on reasonable request. 


\section{Declarations}

\section{Ethics approval and consent to participate}

This study has been performed in accordance with the Declaration of Helsinki and approved by the institutional review board of Shaanxi Provincial People's Hospital, Hospital (No.2017-052), and written informed consent for participation was obtained from all subjects.

\section{Consent for publication}

All involved patients gave their written informed consent to publication.

\section{Competing interests}

The authors declare that they have no competing interests.

\section{Author details}

'Department of Orthopaedics, Shaanxi Provincial People's Hospital (third affiliated hospital of Xi'an jiaotong University), No. 256, Youyi West Road, Xi'an, China. ${ }^{2}$ Shaanxi Provincial Key Laboratory of Infection and Immune Diseases, Shaanxi Provincial People's Hospital (third affiliated hospital of Xi'an jiaotong University), No. 256, Youyi West Road, Xi'an, China.

Received: 3 March 2021 Accepted: 15 November 2021 Published online: 04 December 2021

\section{References}

1. Meier M, Zingde S, Steinert A, Kurtz W, Koeck F, Beckmann J. What is the possible impact of high variability of distal femoral geometry on TKA? A CT data analysis of 24042 knees. Clin Orthop Relat Res. 2019;477(3):561-70

2. Lu F, Sun X, Wang W, Zhang Q, Guo W. Anthropometry of the medial femoral condyle in the Chinese population: the morphometric analysis to design unicomparmental knee component. BMC Musculoskelet Disord. 2021;22(1):95.

3. Katchy AU, Agu AU, Esom E, Ikele IT, Okeke MA, Ugwu AU. The morphometric analysis of the male cadaveric native knees of the ethnic igbos of South East Nigeria and its implication in total knee replacement. Niger J Clin Pract. 2020;23(7):1013-21.

4. Zhang YJ, Chen YX, Qiang MF, Zhang K, Li HB, Jiang YC, et al. Morphometry of the tibial plateau at the surface and resected levels. J Arthroplasty. 2017;32(8):2563-7.

5. Karimi E, Zandi R, Norouzian M, Birjandinejad A. Correlation of anthropometric measurements of proximal tibia in iranian knees with size of current tibial implants. Arch Bone Jt Surg. 2019;7(4):339-45.

6. Wemedke GC, Harris IA, Houang MT, Seeto BG, Chen DB, MacDessi SJ. Comparison of tibial bone coverage of 6 knee prostheses: a magnetic resonance imaging study with controlled rotation. J Orthop Surg (Hong Kong). 2012;20(2):143-7.

7. Cheng FB, Ji XF, Lai Y, Feng JC, Zheng WX, Sun YF, et al. Three dimensional morphometry of the knee to design the total knee arthroplasty for Chinese population. Knee. 2009;16:341-7.

8. Du Z, Chen S, Yan M, Yue B, Wang Y. Differences between native and prosthetic knees in terms of crosssectional morphology of the femoral trochlea: a study based on three-dimensional models and virtual total knee arthroplasty. BMC Musculoskelet Disord. 2017;18(1):166.

9. Loures FB, de Araújo Góes RF, de Sousa EB, Cavanellas N, Barretto JM, Tamaoki MJS, et al. Intraoperative morphometric study of distal femur in Brazilian patients undergoing total knee arthroplasty. PLoS One. 2020;15(5):e0233715.

10. Yang B, Song CH, Yu JK, Yang YQ, Gong X, Chen LX, et al. Intraoperative anthropometric measurements of tibial morphology: comparisons with the dimension of current tibial implants. Knee Surg Sports Traumatol Arthosc. 2014;22(12):2924-30.
11. Incavo SJ, Ronchetti PJ, Howe JG, Tranowski JP. Tibial plateau coverage in total knee arthroplasty. Clin Orthop Relat Res. 1994;299:81-5.

12. Huang $\mathrm{CH}$, Cheng CK, Liau JJ, Lee YM. Morphometrical comparison between the resected surfaces in osteoarthritic knees and Porous-coated Anatomic knee prosthesis. J Musculoskelet Res. 2000;4(1):39-46.

13. Singh JA, Vessely MB, Harmsen WS, Schleck CD, Melton LJ, Kurland RL, et al. A population based study of trends in the use of total hip and TKA, 1969-2008. Mayo Clin Proc. 2010;85:898-904.

14. Yang B, Yu J, Xi G, Lianxu C, Wang Y, Wang J, et al. Sex, age, and annual incidence of primary total knee arthroplasty: a university affiliated hospital survey of 3118 Chinese patients. Chin Med J. 2012;125:3952-5.

15. Asano T, Akagi M, Nakamura T. The functional flexion-extension axis of the knee corresponds to the surgical epicondylar axis: in vivo analysis using a biplanar image-matching technique. J Arthroplasty. 2005;20(8):1060-7.

16. Kirby H, John R, Kenneifi G, Joseph M, Joseph M, Tim H, et al. Anthropometric measurements of the human knee: correlation to the sizing of current knee arthroplasty systems. J Bone Joint Surg Am. 2003;85-A(Suppl 4):115-22.

17. Puthumanapully PK, Harris SJ, Leong A, Cobb JP, Amis AA, Jeffers J. A morphometric study of normal and varus knees. Knee Surg Sports Traumatol Arthrosc. 2014;22(12):2891-9.

18. Amis AA. Biomechanics of high tibial osteotomy. Knee Surg Sports Traumatol Arthrosc. 2013;21:197-205.

19. Hafez MA, Sheikhedrees SM, Saweeres ES. Anthropometry of Arabian arthritic knees: comparison to other ethnic groups and implant dimensions. J Arthroplasty. 2016;31(5):1109-16.

20. Lim HC, Bae JH, Yoon JY, Kim SJ, Kim JG, Lee JM. Gender differences of the morphology of the distal femur and proximal tibia in a Korean population. Knee. 2012;20(1):26-30.

21. Loures FB, de Araújo Góes RF, da Palma IM, Labronici PJ, Granjeiro JM, Olej B. Anthropometric study of the knee and its correlation with the size of three implants available for arthroplasty. Rev Bra Ortop. 2016;51(3):282-9.

22. Kwak DS, Surendran S, Pengatteeri YH, Park SE, Choi KN, Gopinathan $P$, et al. Morphometry of the proximal tibia to design the tibial component of total knee arthroplasty for the Korean population. Knee. 2007;14(4):295-300.

23. Urabe K, Miura H, Kuwano T, Matsuda S, Nagamine R, Sakai S, et al. Comparison between the shape of resected femoral sections and femoral prostheses used in total knee arthroplasty in Japanese patients: simulation using three-dimensional computed tomography. J Knee Surg. 2003;16(1):27-33.

24. Vaidya SV, Ranawat CS, Aroojis A, Laud NS. Anthropometric measurements to design total knee prostheses for the Indian population. J Arthroplasty. 2000;15(1):79-85.

25. Charlton WP, St John TA, Ciccotti MG, Harrison N, Schweitzer M. Differences in femoral notch anatomy between men and women. A magnetic resonance imaging study. Am J Sports Med. 2002;30:329-33.

26. Bing Y, Kartik MV, Songtao A, Tang T, Rubash HE, Li G. Differences of knee anthropometry between Chinese and White men and women. J Arthroplasty. 2011;26(1):124-30.

27. Kim TK, Phillips M, Bhandari M, Watson J, Malhotra R. What differences in morphologic features of the knee exist among patients of various races? A systematic review. Clin Orthop Relat Res. 2017:475(1):170-82.

28. Miyatake N, Sugita T, Aizawa T, Sasaki A, Maeda I, Kamimura M, et al. Comparison of intraoperative anthropometric measurements of the proximal tibia and tibial component in total knee arthroplasty. J Orthop Sci. 2016;21(5):635-9.

29. Iorio R, Kobayashi S, Healy WL, Cruz Al Jr, Ayers ME. Primary posterior cruciate-retaining total knee arthroplasty: a comparison of American and Japanese cohorts. J Surg Orthop Adv. 2007;16(4):164-70.

\section{Publisher's Note}

Springer Nature remains neutral with regard to jurisdictional claims in published maps and institutional affiliations. 